\title{
Aetiology, Clinical Presentation, and Outcome of Meningitis in Patients Coinfected with Human Immunodeficiency Virus and Tuberculosis
}

\author{
Smita Bhagwan and Kogieleum Naidoo \\ Centre for the AIDS Programme of Research in South Africa (CAPRISA), Doris Duke Medical Research Institute (2nd Floor), \\ Nelson R. Mandela School of Medicine, University of KwaZulu-Natal, Private Bag X7, Congella, Durban 4013, South Africa \\ Correspondence should be addressed to Smita Bhagwan, smita.bhagwan@yahoo.com
}

Received 4 October 2011; Accepted 10 November 2011

Academic Editor: Glenda Gray

Copyright ( $) 2011$ S. Bhagwan and K. Naidoo. This is an open access article distributed under the Creative Commons Attribution License, which permits unrestricted use, distribution, and reproduction in any medium, provided the original work is properly cited.

We conducted a retrospective review of confirmed HIV-TB coinfected patients previously enrolled as part of the SAPiT study in Durban, South Africa. Patients with suspected meningitis were included in this case series. From 642 individuals, 14 episodes of meningitis in 10 patients were identified. For 8 patients, this episode of meningitis was the AIDS defining illness, with cryptococcus (9/14 episodes) and tuberculosis (3/14 episodes) as the commonest aetiological agents. The combination of headache and neck stiffness $(78.6 \%)$ was the most frequent clinical presentation. Relapsing cryptococcal meningitis occurred in 3/7 patients. Mortality was 70\% (7/10), with 4 deaths directly due to meningitis. In an HIV TB endemic region we identified cryptococcus followed by tuberculosis as the leading causes of meningitis. We highlight the occurrence of tuberculous meningitis in patients already receiving antituberculous therapy. The development of meningitis heralded poor outcomes, high mortality, and relapsing meningitis despite ART.

\section{Introduction}

Tuberculosis (TB) is the most common opportunistic infection in patients with Human Immunodeficiency Virus (HIV). The estimated relative risk of HIV-infected individuals developing TB is 20.6 compared to HIV uninfected, in populations with a generalized HIV epidemic [1].

HIV contributes significantly to the overall incidence, prevalence and poorer outcomes of meningitis. There is a predominance of chronic opportunistic meningitides in HIV-infected individuals with a higher risk of mortality and impaired cognition [2-6]. It is assumed that positive TB status would account for a greater proportion of tuberculous meningitis among HIV-TB coinfected patients as opposed to cryptococcal meningitis. However, in patients with advanced HIV infection, cryptococcus is the most common aetiology [2].

With increasing numbers of HIV-TB coinfected patients presenting to health facilities and high mortality related to meningitis, we aim to describe the aetiology, clinical presentation, and outcomes of meningitis in HIV-TB coinfected individuals.

\section{Methods}

We retrospectively reviewed HIV-TB coinfected patients with suspected meningitis. Patients 18 years and older, with confirmed pulmonary TB and HIV, enrolled into the SAPiT study, presenting with suspected meningitis were included in this study. The SAPiT study was a prospective randomized control trial conducted in Durban, South Africa (June 2005July 2008), investigating the optimal timing of antiretroviral therapy (ART) initiation in patients on antituberculous therapy. All patients in the SAPiT study were sputum smear positive for mycobacteria on enrollment. They were randomly assigned to three groups, each group initiating ART at a different stage in tuberculosis therapy: within 4 weeks after the start of tuberculosis therapy (early arm), within 4 weeks 
after the completion of the intensive phase of tuberculosis therapy (post intensive arm), or within 4 weeks of completion of tuberculosis therapy (postcontinuation arm) [7].

Meningitis was suspected in patients who had meningeal symptoms (headache, neck stiffness, photophobia, vomiting) alone or occurring with fever, altered level of consciousness, or focal neurological signs. Data on clinical presentation, investigations, and outcomes were extracted from records at the clinical trial site and hospitals to which patients were admitted. The diagnoses of meningitis were made using a combination of recorded clinical and cerebrospinal fluid (CSF) criteria.

(i) Cryptococcal meningitis: diagnosed based on a positive CSF India ink stain, CSF cryptococcal antigen test or patients initiated on systemic antifungal agents with positive response.

(ii) Tuberculous meningitis: diagnosed if Mycobacterium tuberculosis was isolated in the CSF. In the absence of definitive microbiologic evidence, tuberculous meningitis was diagnosed based on neurological findings in combination with typical CSF alterations (lymphocytic predominance, raised protein, reduced glucose), with exclusion of other aetiologies.

(iii) Acute bacterial meningitis: diagnosed on detection of bacteria by CSF microscopy or culture, or acute clinical presentation with characteristic CSF findings (neutrophil predominance, raised protein, reduced glucose), and prompt response to antibacterial drugs $[2,3]$.

Patients were excluded if records of their diagnostic information were incomplete or unavailable. Recurrent episodes of meningitis that occurred in patients after discharge from hospital during the follow-up period were included.

This was a descriptive observational study using frequencies and percentages to summarize categorical data and means, medians, and ranges to summarize continuous data. The SAPiT trial was approved by the Biomedical Research Ethics Committee at the University of KwaZulu-Natal and the South African government's Medicines Control Council.

\section{Results}

From 642 TB-HIV coinfected patients enrolled, 20 (3.1\%) patients presented with clinical features suggestive of meningitis, with $10(50 \%)$ classified as not having meningitis (two cerebral tuberculomas, one cerebral toxoplasmosis, three with normal CSF, and four with insufficient data to support our case definitions). Fourteen episodes of meningitis that met our case definitions occurred in 10 patients, summarized in Table 1.

Among the patients analyzed, the mean (range) age was 34 (24-48) years and 4 patients were female. The mean (range) BMI was 22.13 (15.9-30.2) kg/m² and median (range) baseline CD4+ cell count was 13 (2-298) cells $/ \mathrm{mm}^{3}$. For 8 patients, the episode of meningitis under study was the first Acquired Immunodeficiency Syndrome (AIDS) illness experienced. Five patients reported a previous history of
TB and 2 reported a previous history of meningitis, both receiving antifungal prophylaxis for cryptococcal meningitis after the first episode.

The most common combination of presenting clinical features was headache and neck stiffness, seen in $11(78.6 \%)$ episodes and the triad of headache, vomiting, and neck stiffness observed in $5(35.7 \%)$ episodes. Only 2 patients presented with fever.

There were nine (9/14) episodes of cryptococcal meningitis (one with disseminated cryptococcal disease, Patient 3 ), three (3/14) episodes of tuberculous meningitis (one concomitantly with suspected neurocysticercosis, patient 9), and two (2/14) cases of meningitis with mixed aetiology (Patient 3, acute bacterial and cryptococcal; Patient 7, cryptococcal and tuberculous).

TB sputum culture testing was done for $8 / 10$ patients with meningitis: 4 of these sputum samples were taken within 6 weeks of the diagnosis of meningitis. All 4 sputum samples were culture positive for TB and occurred in patients with cryptococcal meningitis. There were no cases of drugresistant tuberculosis.

The median number of days (range) to the episode of meningitis from antituberculous therapy initiation was 109 (32-486) and from ART initiation in the 7 patients receiving ART was 70 (7-214) days. All patients who were diagnosed with tuberculous meningitis were already on antituberculous therapy with 2 patients in the last month of the continuation phase. Patients 7 and 8 (Table 1) received steroids as part of tuberculous meningitis management. Four patients (Patients 1, 3, 7, 10) had suspected immune reconstitution inflammatory syndrome (IRIS) based on the temporal association of meningitis to ART initiation.

Of the 10 patients, 7 died, with 4 deaths directly due to meningitis: cryptococcal infection, 1, tuberculosis, 2, and 1 patient with mixed cryptococcal and tuberculous meningitis. From patients receiving ART, four (4/7) died. The case fatality rate for cryptococcal meningitis alone was $11.1 \%(1 / 9)$ and increased to $22.2 \%(2 / 9)$ when cryptococcal meningitis contributed to death as part of a mixed infection. Among the 7 patients with cryptococcal meningitis, 3 had relapsing episodes. The case fatality rate for tuberculous meningitis alone was $66.6 \%(2 / 3)$ and increased to $75 \%(3 / 4)$ when tuberculous meningitis contributed to death as part of a mixed infection.

The distribution of deaths among the SAPiT arms was as follows: 2 deaths in the early arm (1 from meningitis); 2 deaths in the postintensive arm (1 from meningitis); 3 deaths in the postcontinuation arm (2 from meningitis). Three patients died within 3.5 months after the episode of meningitis from other causes, attesting to the advanced immunocompromised state of these patients.

\section{Discussion}

In this first case series evaluating meningitis in confirmed HIV-TB coinfected patients, we demonstrate that the leading cause of meningitis is cryptococcus followed by tuberculosis, which is in keeping with population-based reports from subSaharan Africa investigating meningitis in patients with HIV 


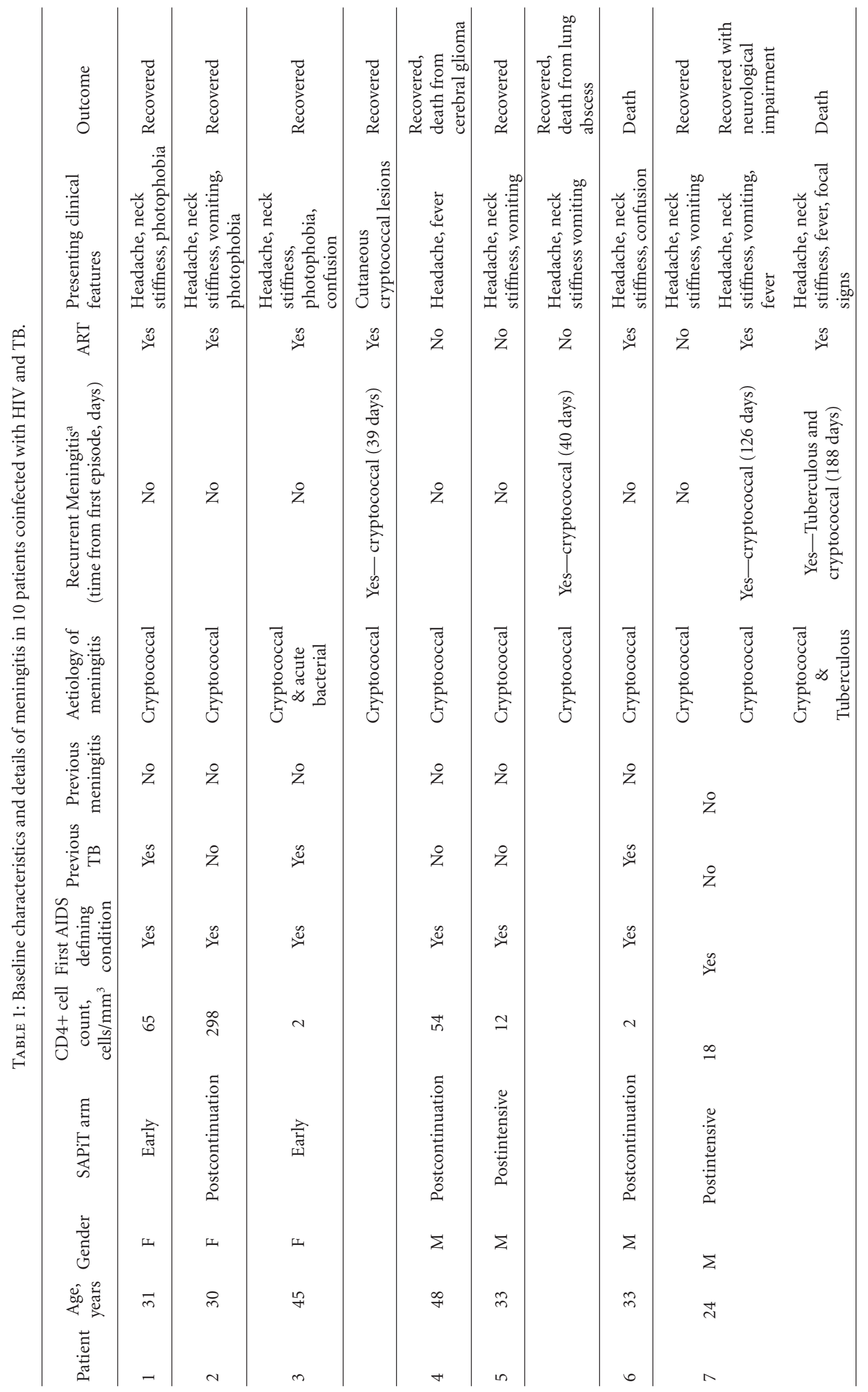




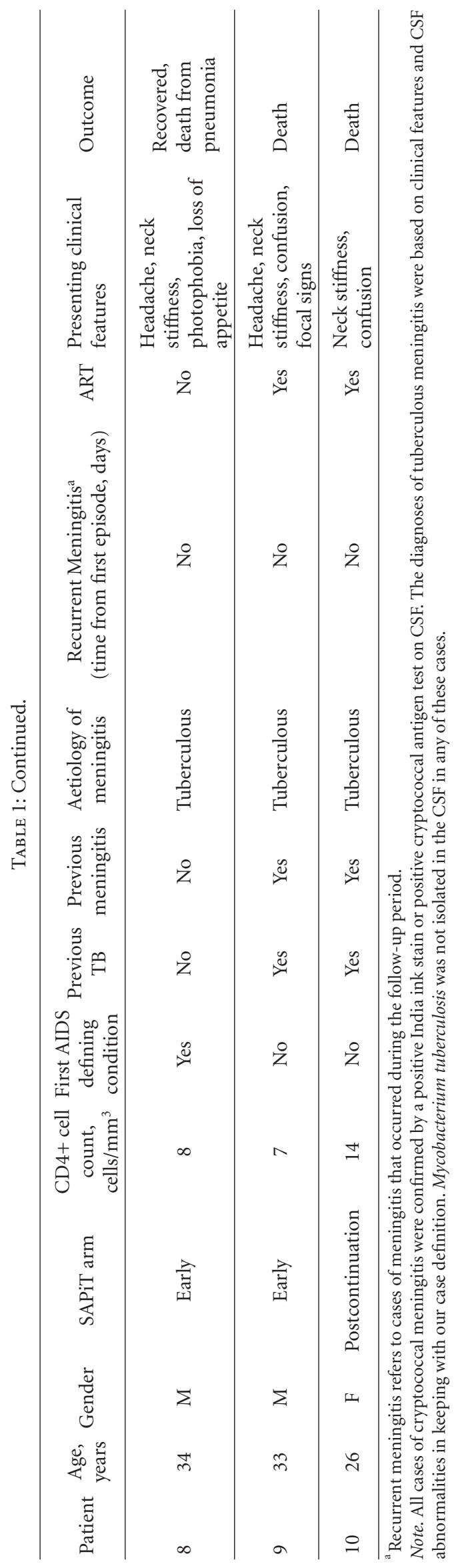


alone [2]. In this sample of coinfected patients, development of meningitis was associated with poor outcomes including relapsing meningitis (30\%) and high mortality (40\%), even in patients already initiated on ART (57\%). Patients in this study were severely immunocompromised with low CD4+ cell counts (median 13 cells $/ \mathrm{mm}^{3}$ ), which could account for the reduced frequency of fever as a presenting complaint and the high mortality and relapse rates.

The burden of tuberculous meningitis has escalated as HIV disease progression increases extrapulmonary tuberculous risk. As established previously [2], the proportion of tuberculous meningitis is commonly underestimated due to its associated diagnostic challenges, specifically the inability to isolate mycobacteria in the CSF $[2,4,8]$. In this study, microbiologic diagnosis of tuberculous meningitis was further compromised as patients had already received several weeks to months of antituberculous therapy. Our study highlighted the important occurrence of tuberculous meningitis in patients already receiving antituberculous therapy which, to our knowledge, has only been investigated once previously, also in Southern Africa [5]. Antituberculous drug resistance and TB-associated IRIS should be principal considerations in such patients. Coinfected patients had poorer outcomes with case fatality rates of $66.7 \%$ compared to a case fatality rate of $30 \%$ from reports of tuberculous meningitis patients without coinfection [4]. This highlights the need to reevaluate management of tuberculous meningitis particularly in patients already exposed to antituberculous therapy. Evaluation of alternate drugs that optimally penetrate the blood brain barrier and the timing and overall benefit of steroids in HIV-associated tuberculous meningitis remains a challenge. Isoniazid and pyrazinamide are first-line antituberculous drugs with good CSF penetration. Patients who develop tuberculous meningitis while in the continuation phase of antituberculous therapy are managed with the retreatment regimen which includes streptomycin. However, injectable aminoglycosides have poor distribution in the CSF. The use of second-line drugs or alternative agents with good CSF penetration such as cycloserine and ethionamide rather than the conventional retreatment regimen needs further investigation $[9,10]$.

The estimated case fatality rate of cryptococcal meningitis in sub-Saharan Africa of $70 \%$ is considerably higher than in our study [11]. However, our sample is small resulting in an unstable estimate. Other contributing factors that could account for this differential mortality include availability of dual systemic antifungal agents, the urban location of our research facility with established systems for rapid triage into appropriate care and supporting laboratory infrastructure.

Three of seven $(42.9 \%)$ patients experienced cryptococcal meningitis relapses, exceeding relapse rates reported in patients with HIV alone (23\%). Contributing factors to the high relapse rate include inadequate clinical and serological screening for cryptococcal disease with use of appropriate prophylaxis, fluconazole resistance, and cryptococcal associated IRIS in patients on ART [12]. The underlying pathophysiologic mechanisms for the higher rates of relapse in coinfected patients needs further study.
We acknowledge several limitations in this study, particularly the small sample size. Patients were admitted to various facilities and were managed by health care workers with differing skills and access to laboratory and pharmacological resources. As this was a retrospective chart review, the quality and completeness of data obtained varied between patients.

Meningitis in HIV-infected individuals can be caused by various aetiological agents [2]. This study demonstrates cryptococcus as the leading cause of meningitis in HIV$\mathrm{TB}$ coinfected patients, highlighting the occurrence of meningitis due to causative agents other than TB, in HIVinfected patients with sputum culture positive TB. However, clinicians should be wary that tuberculous meningitis can occur in patients already receiving antituberculous therapy. Coinfected patients who developed cryptococcal meningitis demonstrated a higher relapse rate. The mortality from meningitis in coinfected patients is high, particularly in those with tuberculous meningitis, and even in patients on antiretroviral therapy, emphasizing the necessity for timely diagnosis of HIV and meningitis and to establish optimum timing of ART in patients with central nervous system infections. Our data supports the need for further research into strategies for early detection of meningitis, improved diagnostic methods for tuberculous meningitis, and evaluation of more effective drug regimens to improve the prognosis of HIV associated meningitis.

\section{Conflict of Interests}

There were no conflicts of interests.

\section{Authors' Contribution}

Both authors contributed significantly in study conception and design, acquisition, analysis, and interpretation of data as well as paper preparation.

\section{Acknowledgments}

This work was supported by the National Institutes of Health, Fogarty International Center through the International Clinical Research Scholars and Fellows Program at Vanderbilt University [R24 TW007988]. The authors thank the reviewer of their paper, Dr Vinod Patel, and Nonhlanhla Yende for her statistical advice. They thank their mentors, Professor Quarraisha Abdool Karim and Professor Salim Abdool Karim, for scientific mentorship. The investigators were supported by the Columbia UniversitySouthern African Fogarty AIDS International Training and Research Program (AITRP) funded by the Fogarty International Center, National Institutes of Health (Grant no. D43TW00231). CAPRISA was part of the Comprehensive International Program of Research on AIDS (CIPRA) and is supported by the National Institute of Allergy and Infectious Disease (NIAID), National Institutes of Health (NIH) and the US Department of Health and Human Services (Grant no. AI51794), which established the research infrastructure 
to conduct the SAPiT trial, including the data management, laboratory and pharmacy core. The US President's Emergency Plan for AIDS Relief (PEPfAR) funded the care of all the participants in the trial. The authors gratefully acknowledge the participants in the study.

\section{References}

[1] S. D. Lawn and G. Churchyard, "Epidemiology of HIVassociated tuberculosis," Current Opinion in HIV and AIDS, vol. 4, no. 4, pp. 325-333, 2009.

[2] J. N. Jarvis, G. Meintjes, A. Williams, Y. Brown, T. Crede, and T. S. Harrison, "Adult meningitis in a setting of high HIV and TB prevalence: Findings from 4961 suspected cases," BMC Infectious Diseases, vol. 10, article 67, 2010.

[3] A. Bergemann and A. S. Karstaedt, "The spectrum of meningitis in a population with high prevalence of HIV disease," Quarterly Journal of Medicine, vol. 89, no. 7, pp. 499-504, 1996.

[4] G. E. Thwaites, N. D. Bang, N. H. Dung et al., "The influence of HIV infection on clinical presentation, response to treatment and outcome in adults with tuberculous meningitis," Journal of Infectious Diseases, vol. 192, no. 12, pp. 2134-2141, 2005.

[5] E. Silber, P. Sonnenberg, K. C. Ho et al., "Meningitis in a community with a high prevalence of tuberculosis and HIV infection," Journal of the Neurological Sciences, vol. 162, no. 1, pp. 20-26, 1999.

[6] S. M. Katrak, P. K. Shembalkar, S. R. Bijwe, and L. D. Bhandarkar, "The clinical, radiological and pathological profile of tuberculous meningitis in patients with and without human immunodeficiency virus infection," Journal of the Neurological Sciences, vol. 181, no. 1-2, pp. 118-126, 2000.

[7] S. S. Abdool Karim, K. Naidoo, A. Grobler et al., "Timing of initiation of antiretroviral drugs during tuberculosis therapy," New England Journal of Medicine, vol. 362, no. 8, pp. 697-706, 2010.

[8] A. I. Bhigjee, R. Padayachee, H. Paruk, K. D. HallwirthPillay, S. Marais, and C. Connoly, "Diagnosis of tuberculous meningitis: clinical and laboratory parameters," International Journal of Infectious Diseases, vol. 11, no. 4, pp. 348-354, 2007.

[9] World Health Organization, "Treatment of tuberculosis: guidelines, 4th edition,” (WHO/HTM/TB/2009.420), 2009, http://www.who.int/tb/publications/cds_tb_2003_313/en/.

[10] P. R. Donald, "Cerebrospinal fluid concentrations of antituberculosis agents in adults and children," Tuberculosis, vol. 90, no. 5, pp. 279-292, 2010.

[11] B. J. Park, K. A. Wannemuehler, B. J. Marston, N. Govender, P. G. Pappas, and T. M. Chiller, "Estimation of the current global burden of cryptococcal meningitis among persons living with HIV/AIDS," AIDS, vol. 23, no. 4, pp. 525-530, 2009.

[12] J. N. Jarvis, G. Meintjes, Z. Williams, K. Rebe, and T. S. Harrison, "Symptomatic relapse of HIV-associated cryptococcal meningitis in South Africa: the role of inadequate secondary prophylaxis.," South African Medical Journal, vol. 100, no. 6, pp. 378-382, 2010. 


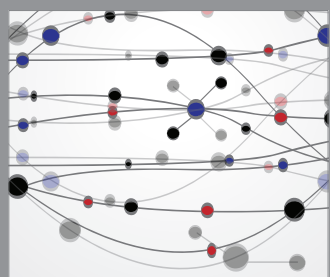

The Scientific World Journal
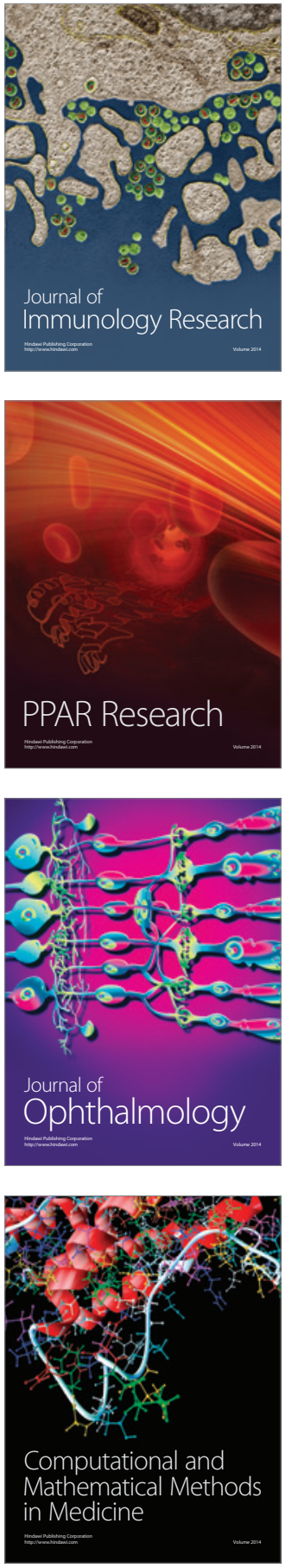

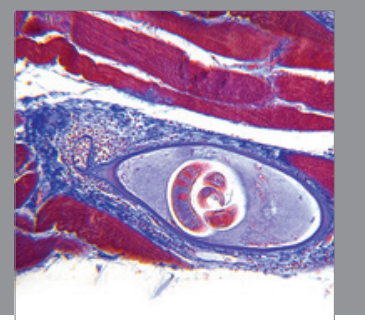

Gastroenterology

Research and Practice
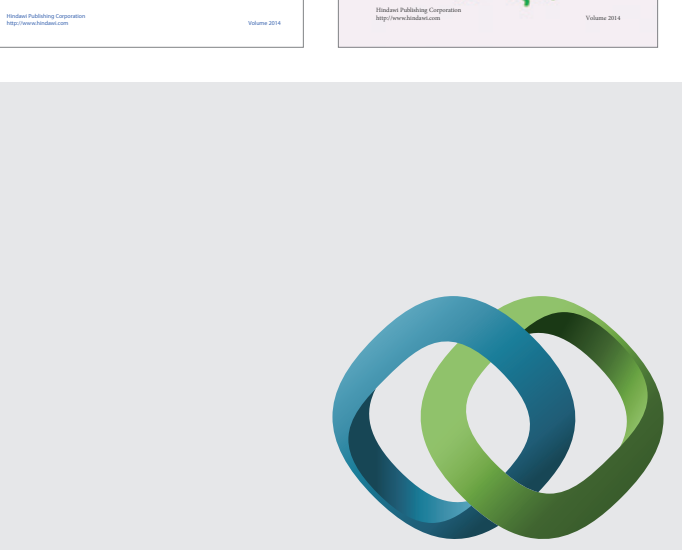

\section{Hindawi}

Submit your manuscripts at

http://www.hindawi.com
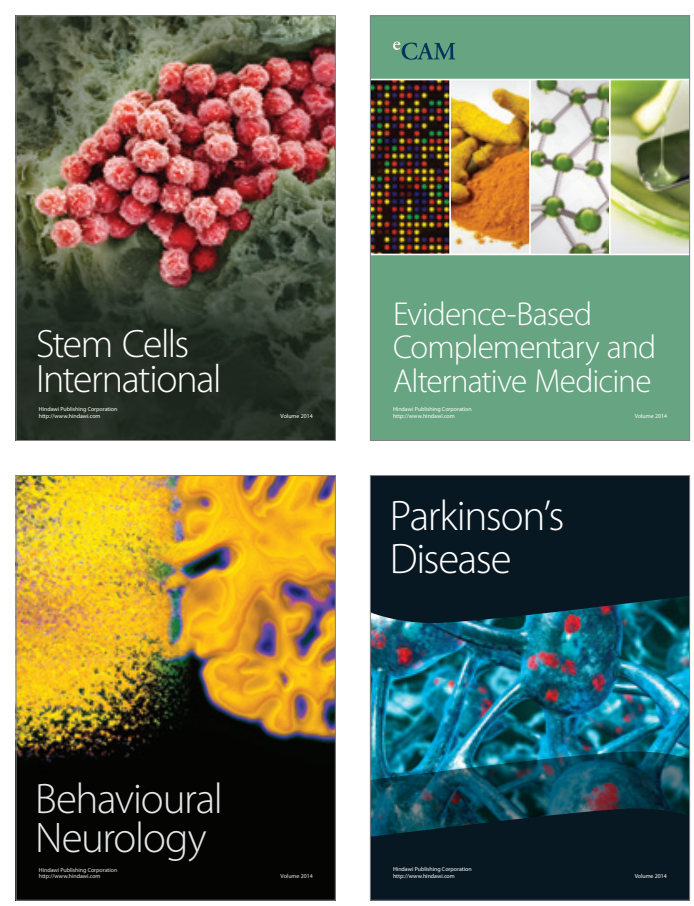

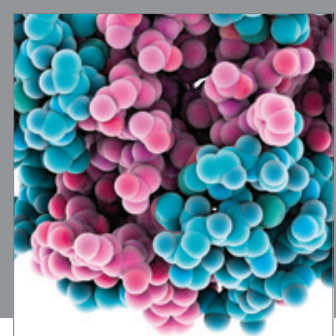

Journal of
Diabetes Research

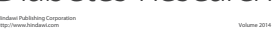

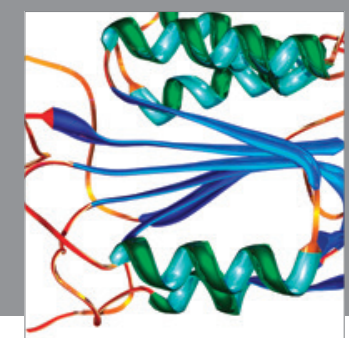

Disease Markers
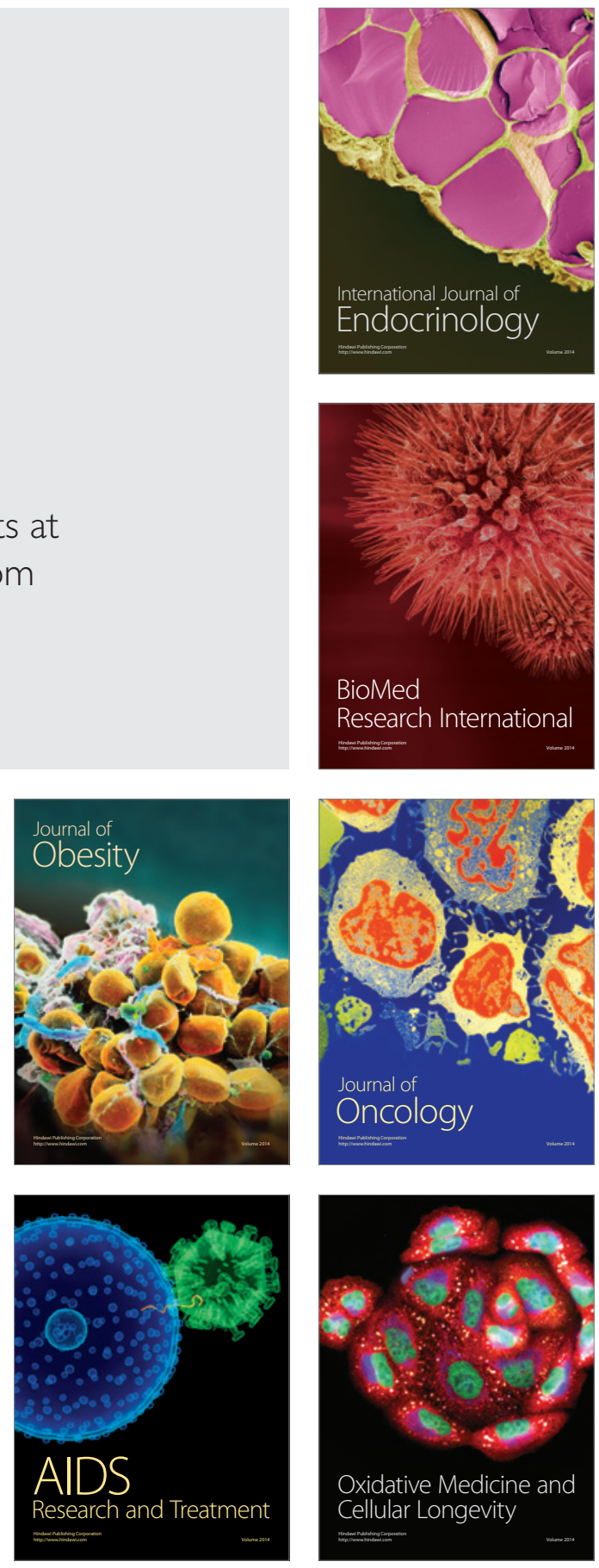